\title{
Structured reporting of brain MRI following mechanical thrombectomy in acute ischemic stroke patients
}

\author{
Sebastian Mönch ${ }^{1,2^{*}}$, Tiberiu Andrisan ${ }^{1}$, Kathleen Bernkopf ${ }^{3}$, Benno Ikenberg $^{3}$, Benjamin Friedrich? , \\ Claus Zimmer ${ }^{1}$ and Dennis M. Hedderich ${ }^{1}$
}

\begin{abstract}
Background: To compare the quality of free-text reports (FTR) and structured reports (SR) of brain magnetic resonance imaging (MRI) examinations in patients following mechanical thrombectomy for acute stroke treatment.

Methods: A template for SR of brain MRI examinations based on decision trees was designed and developed in house and applied to twenty patients with acute ischemic stroke in addition to FTR. Two experienced stroke neurologists independently evaluated the quality of FTR and SR regarding clarity, content, presence of key features, information extraction, and overall report quality. The statistical analysis for the differences between FTR and SR was performed using the Mann-Whitney U-test or the Chi-squared test.

Results: Clarity $(p<0.001)$, comprehensibility $(p<0.001)$, inclusion of relevant findings $(p=0.016)$, structure $(p=0.005)$, and satisfaction with the content of the report for immediate patient management $(p<0.001)$ were evaluated significantly superior for the SR by both neurologist raters. One rater additionally found the explanation of the patient's clinical symptoms $(p=0.003)$, completeness $(p<0.009)$ and length $(p<0.001)$ of SR to be significantly superior compared to FTR and stated that there remained no open questions, requiring further consultation of the radiologist $(p<0.001)$. Both neurologists preferred SR over FTR.
\end{abstract}

Conclusions: The use of SR for brain magnetic resonance imaging may increase the report quality and satisfaction of the referring physicians in acute ischemic stroke patients following mechanical thrombectomy.

Trial registration Retrospectively registered.

Keywords: Structured reporting, Quality assessment, Magnetic resonance imaging, Acute ischemic stroke, Mechanical thrombectomy

\section{Background}

Ischemic stroke is a leading cause of disability and mortality worldwide [1]. Recently, mechanical thrombectomy (MT) has been proven to be highly beneficial for clinical

\footnotetext{
*Correspondence: moench.s@gmx.net

${ }^{1}$ Department of Diagnostic and Interventional Neuroradiology, Klinikum Rechts Der Isar, School of Medicine, Technical University Munich, Ismaninger Straße 22, 81675 Munich, Germany

Full list of author information is available at the end of the article
}

outcomes of acute ischemic stroke patients (AIS) with an underlying large vessel occlusion [2-6]. In addition to this revolution in AIS therapy, great efforts have been put into individualizing rehabilitation measures following stroke unit or intensive care treatment [7]. In addition to the clinical assessment, specific knowledge about the affected brain regions and possible complications, such as hemorrhage or brain edema, is essential for clinical decision-making and thus for the patient's prognosis and grade of disability. Magnetic resonance imaging 
(MRI) of the brain is a mainstay in neuroradiological practice and is essential for the assessment of stroke. To date, the vast majority of these radiology reports are produced in free-text report (FTR) format, describing narrative reports which were typed manually or dictated into voice recording systems. FTR are known to be associated with excessive variability in language, clarity, and content [8]. Thus, report quality may be reduced, making it potentially more difficult for referring clinicians to identify key points necessary for patient care. Structured reports (SR), are typically based on systematic checklist and decision tree options using specifically designed templates and have gained much attention recently. By minimizing shortcomings of FTR disadvantages and serving as an ideal basis for artificial intelligence applications, they have been introduced into clinical practice lately $[8,9]$. For example, SR of brain MRI examinations where shown to increase the rate of included disease-relevant findings in Multiple Sclerosis patients and were preferred by clinicians [10]. We know from previous studies that SR increases report completeness and attention to detail $[11,12]$. As stated above, detailed and clear descriptions of affected brain regions are of high clinical interest in subacute stroke patients. Thus, we hypothesized that SR would be favorable over FTR in patients after MT. We addressed this question in a single-center, retrospective study.

\section{Methods}

\section{Study design}

In this monocentric, retrospective study, consecutive AIS patients from daily clinical routine who had been treated with MT and had an MRI report of the brain from two neuroradiologists (S.M. or D.M.H.) three to five days after this procedure were included from 08/2017 until 03/2018. Informed consent was waived due to the retrospective nature of the study by the ethics committee of the Technical University of Munich. All procedures were approved by the ethics committee of the Technical University of Munich (application number 56/20 S-KH). All methods were carried out in accordance with the Declaration of Helsinki.

\section{Endovascular Intervention}

Patients were eligible for MT if CT angiography (CTA) confirmed a large vessel occlusion of the internal carotid artery, the middle cerebral artery or the basilar artery. Parenchymal infarction was limited to an Alberta Stroke Program Early CT Score (ASPECTS) of $>5$ in middle cerebral artery strokes. No age or perfusion selection was applied within the timeframe of $6 \mathrm{~h}$. Patients beyond the time window of $6 \mathrm{~h}$ since symptom onset or with unknown/wake-up symptom onset were selected by a target mismatch on CT Perfusion according to local standard operating procedures. For basilar artery occlusions no time window was applied. After a femoral access was established a stent-retriever based MT was performed. Hemorrhagic transformation of ischemic infarct were defined according to ECASS II-classification [13]. Successful recanalization was defined as an extended Thrombolysis in cerebral infarction score of greater than or equal to $2 \mathrm{~b}$. Procedure related complications were defined as arterial dissection, intracranial vessel perforation or subarachnoid hemorrhage on control CT imaging. Intravenous thrombolysis was performed by weight adapted tissue plasminogen activator according to current guidelines.

\section{Brain MRI}

The brain MRI scanning protocol included axial fluid attenuation inversion recovery (FLAIR), axial T2*, axial T2, and 3D arterial time-of-flight MR angiography (art. TOF) and axial diffusion weighted imaging (DWI) sequences (Table 1). All scans were performed using a 3.0 T MRI system (Philips Achieva dStream 3.0 T, Philips Healthcare, Best, Netherlands) equipped with a 32-channel phased array head coil (Philips) for signal reception.

\section{Structured reporting template for stroke MRI}

The content and design of the SR template was performed by neuroradiologists (S.M., D.M.H.). The SR template was developed in house. In principle, this software allows the radiologist to select check boxes containing subitems based on decision trees (excerpt of this template shown in Fig. 1). These clickable decisions are then automatically transferred into predefined text phrases. The SR template additionally offered the option to manually add sentences in dedicated free-text fields.

Next to the appearance on MRI sequences the details on infarct location and extent are subitems to be selected by the radiologist in order for the software to concomitantly generate the report. Abbreviations:

Table 1 Sequence parameter specifications

\begin{tabular}{lclll}
\hline Sequence & TE $(\mathbf{m s})$ & TR $(\mathbf{m s})$ & TI $(\mathbf{m s})$ & AT $(\mathbf{s})$ \\
\hline Ax. FLAIR & 140.0 & 12,000 & 2850.0 & 180 \\
Ax. T2 & 80.0 & 3200 & $/$ & 166 \\
Ax. T2* & 16.1 & 858 ("shortest") $^{*}$ & $/$ & 147 \\
3D art. TOF & 3.5 & 25 & $/$ & 360 \\
Ax. DWI & 55.0 & 10,126 & $/$ & 366 \\
\hline
\end{tabular}

$T E$ time to echo, $T R$ time to repeat, $T I$ inversion time, $A T$ acquisition time, / not applicable, $A x$. axial, FLAIR fluid attenuated inversion recovery, art. TOF arterial time-of-flight MR angiography, DWI diffusion weighted imaging 


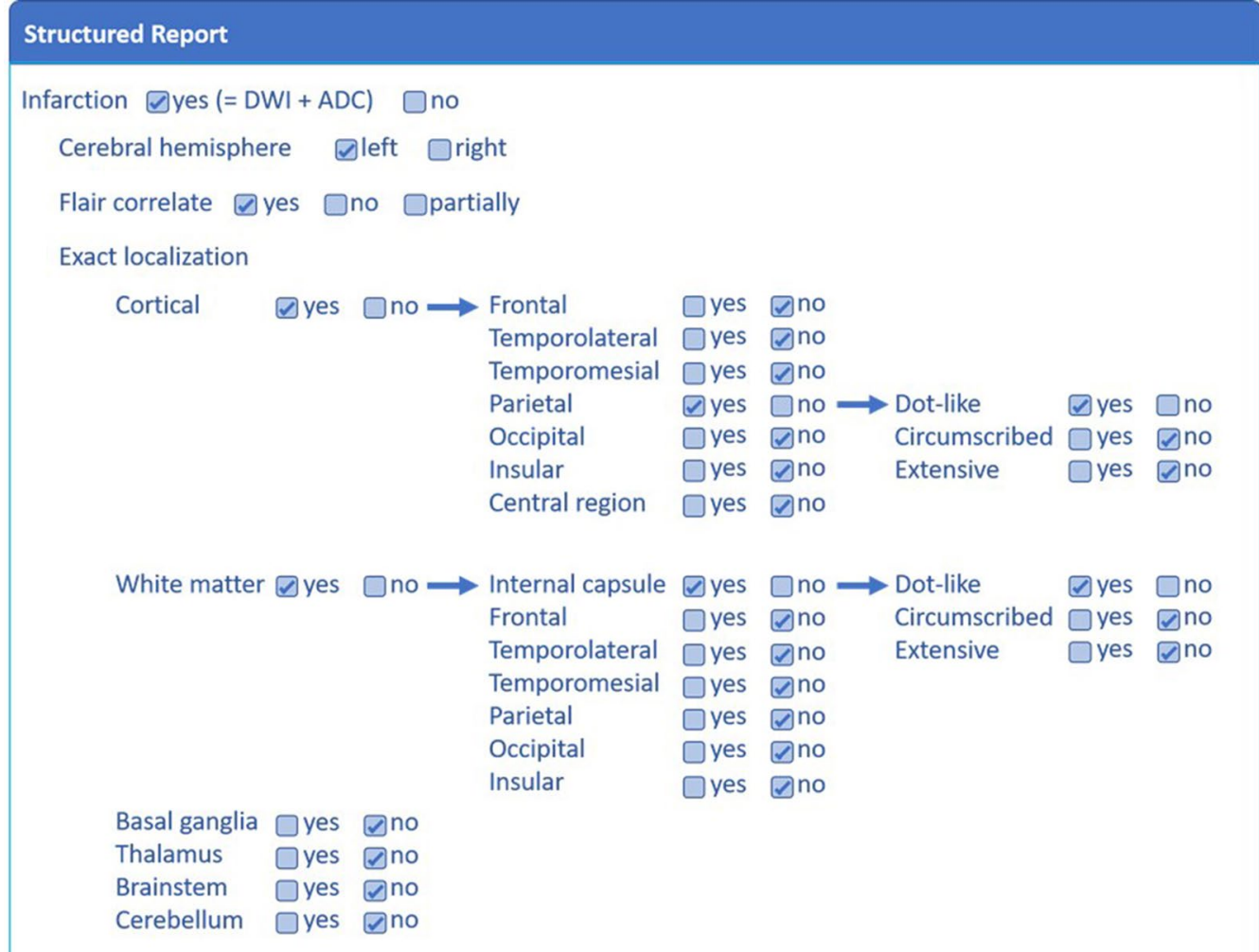

Fig. 1 Conceptual example of the clickable decision tree of the structured reporting template

$\mathrm{DWI}=$ Diffusion weighted imaging, $\mathrm{ADC}=$ Apparent diffusion coefficient, FLAIR = Fluid-attenuated inversion recovery.

The SR consisted of a findings section and an impressions section. In the findings section, the radiologist had to provide information regarding initially occluded vessel, presence of stenting procedures, intravenous lysis administration, recanalization success, MRI sequences, infarction (diffusion restriction, mismatch between diffusions weighted image and FLAIR image, extent (dot-like, circumscribed, extensive), localization (frontal, temporolateral, temporomesial, parietal, occipital, insular, central region), affection (cortex, white matter, basal ganglia, thalamus, cerebellum or brain stem), complications (e.g. intracranial hemorrhage, hemorrhagic transformation of ischemic infarct, infarct swelling, obstructive hydrocephalus, midline shift, herniation), status of recanalized vessel (stenosis, persisting occlusion) and presence of other occluded vessels, microangiopathy, and older infarcts. This information was then automatically transferred and summarized in the impression section.

\section{Free-text reports}

As FTR, the original, already existing report generated manually or by using a voice dictating system were used. All FTRs were produced by the same radiologist as the SR and proofread by a neuroradiology consultant in clinical routine.

\section{Report evaluation}

The reports and MR images were reviewed using a standard clinical PACS (Picture Archiving and Communication System) workstation (IDS7, Sectra AB, Linköping, Sweden). The images were then randomly ordered and pseudonymized, blinded for patient data including the original FTR, date of the examination. Two neuroradiologists, with six years (D.M.H.) and three years (S.M.) of experience in the interpretation of brain MRI examinations independently reviewed all images and established a SR for each patient using the described SR template. Afterwards, two neurologists, with seven (B.I.) and five (K.B.) years of experience evaluated and compared the original FTR and the newly generated SR. These two neurologists were not involved in the primary study design. 
The quality of these reports was then evaluated by the two neurologists on a Likert scale from 1 to 10 or from 1 to 5 depending on the question (Fig. 2). The statistical analysis for the differences between FTR and SR was performed using the Mann-Whitney U-test or the Chisquared test. Statistical significance was assumed for $\mathrm{p}<0.05$.

\section{Statistical analysis}

The statistical analysis was performed with the Statistical Package for Social Sciences v. 25 (SPSS, version 25,
Chicago, IL, USA) and with Excel 2013 software (Microsoft, Redmond, WA, USA).

\section{Results}

In this study, a total of 40 pseudonymized reports, 20 FTRs and 20 corresponding SRs, of 20 patients with AIS who had received an MRI of the brain three to five days after MT were included in the present study (Table 2).

The questionnaire evaluation results of two neurologist experienced in treating AIS patients are depicted in Fig. 2. Both raters significantly favored the SR over the FTR

\section{Please answer the following questions on a scale from 1 („does not apply at all”) to 10} („perfectly applies“)

\begin{tabular}{|l|l|}
\hline 1. The report is clear & $\square$ 1 $\square 2 \square 3 \square 4 \square 5 \square 6 \square 7 \square 8 \square 9 \square 10$ \\
\hline 2. The report is clearly comprehensible & $\square 1 \square 2 \square 3 \square 4 \square 5 \square 6 \square 7 \square 8 \square 9 \square 10$ \\
\hline 3. The report has an appropriate length & $\square 1 \square 2 \square 3 \square 4 \square 5 \square 6 \square 7 \square 8 \square 9 \square 10$ \\
\hline $\begin{array}{l}\text { 4. The report allows to extract relevant } \\
\text { findings easily }\end{array}$ & $\square 1 \square 2 \square 3 \square 4 \square 5 \square 6 \square 7 \square 8 \square 9 \square 10$ \\
\hline $\begin{array}{l}\text { 5. The structure of the report helps in } \\
\text { finding relevant information quickly }\end{array}$ & $\square 1 \square 2 \square 3 \square 4 \square 5 \square 6 \square 7 \square 8 \square 9 \square 10$ \\
\hline $\begin{array}{l}\text { 6. The report contains all relevant } \\
\text { information }\end{array}$ & $\square 1 \square 2 \square 3 \square 4 \square 5 \square 6 \square 7 \square 8 \square 9 \square 10$ \\
\hline $\begin{array}{l}\text { 7. The report allows an explanation of the } \\
\text { patient's clinical symptoms }\end{array}$ & $\square 1 \square 2 \square 3 \square 4 \square 5 \square 6 \square 7 \square 8 \square 9 \square 10$ \\
\hline $\begin{array}{l}\text { 8. The report contains all information } \\
\text { necessary for the subsequent patient } \\
\text { management (e.g. restart with } \\
\text { anticoagulation) }\end{array}$ & $\square 1 \square 2 \square 3 \square 4 \square 5 \square 6 \square 7 \square 8 \square 9 \square 10$ \\
\hline $\begin{array}{l}\text { 9. I have no open questions, a further } \\
\text { consultation with the radiologist is not } \\
\text { necessary }\end{array}$ & $\square 1 \square 2 \square 3 \square 4 \square 5 \square 6 \square 7 \square 8 \square 9 \square 10$ \\
\hline $\begin{array}{l}\text { 10. The report explains the MR images } \\
\text { well }\end{array}$ & $\square 1 \square 2 \square 3 \square 4 \square 5 \square 6 \square 7 \square 8 \square 9 \square 10$ \\
\hline
\end{tabular}

11. Please evaluate the overall quality of the report on a scale from 1 to 5 :

(1: insufficient; 2 : low quality; 3 : medium quality; 4 : high quality; 5 : excellent quality)

$$
\square 1 \quad \square 2 \quad \square 3 \quad \square 4 \quad \square 5
$$

12. Favored report type:

$$
\text { structured }
$$

free text

Fig. 2 Questionnaire for neurologists 
Table 2 Baseline patient characteristics as well as procedure metrics and patient outcome

\begin{tabular}{|c|c|}
\hline Gender [\% female; $\mathrm{n}]$ & $45(9 / 20)$ \\
\hline Age [years] & $74.2 \pm 11.1$ \\
\hline Atrial fibrillation [\%; n] & $20(4 / 20)$ \\
\hline Hypertension [\%; n] & $25(5 / 20)$ \\
\hline Smoker [\%; n] & $85(17 / 20)$ \\
\hline Diabetes [\%; n] & $25(5 / 20)$ \\
\hline Hyperlipidemia[\%; n] & $40(8 / 20)$ \\
\hline Wake-up stroke & $25(5 / 20)$ \\
\hline NIHSS on admission & $12.8 \pm 6.8$ \\
\hline \multicolumn{2}{|l|}{ Vessel occlusion [\%; n] } \\
\hline Middle cerebral artery & $70(14 / 20)$ \\
\hline Distal internal carotid artery & $20(4 / 20)$ \\
\hline Basilar artery & $10(2 / 20)$ \\
\hline Intravenous thrombolysis [\%; n] & $45(9 / 20)$ \\
\hline NIHSS after intervention [\%; n] & $6.6 \pm 7.1$ \\
\hline Time to recanalization [min] & $207 \pm 73$ \\
\hline Successful recanalization [\%; n] & $85(17 / 20)$ \\
\hline Procedure related complications [\%; n] & $0(0 / 20)$ \\
\hline Intracranial hemorrhages [\%; n] & $0(0 / 20)$ \\
\hline \multicolumn{2}{|l|}{ Hemorrhagic transformation } \\
\hline Hemorrhagic infarction type $1[\% ; n]$ & $20(4 / 20)$ \\
\hline Hemorrhagic infarction type 2 [\%; n] & $5(1 / 20)$ \\
\hline Parenchymal hemorrhage type $1[\% ; n]$ & $10(2 / 20)$ \\
\hline Parenchymal hemorrhage type $2[\% ; n]$ & $0(0 / 20)$ \\
\hline
\end{tabular}

Table 3 Results of report quality ratings FTR versus SR

\begin{tabular}{|c|c|c|c|c|c|c|}
\hline \multirow[t]{2}{*}{ Questions } & \multicolumn{2}{|l|}{ Rater 1} & \multirow[t]{2}{*}{$p$ value } & \multicolumn{2}{|l|}{ Rater 2} & \multirow[t]{2}{*}{$p$ value } \\
\hline & FTR & SR & & FTR & SR & \\
\hline 1. The report is clear & $8.05 \pm 0.51$ & $9.05 \pm 0.22$ & $<0.001^{*}$ & $6.80 \pm 1.28$ & $8.30 \pm 0.66$ & $<0.001^{*}$ \\
\hline 2. The report is clearly comprehensible & $8.50 \pm 0.61$ & $9.10 \pm 0.44$ & $0.001^{*}$ & $7.35 \pm 0.81$ & $8.20 \pm 0.83$ & $0.001^{*}$ \\
\hline 3. The report has an appropriate length & $8.60 \pm 0.50$ & $8.40 \pm 0.60$ & 0.293 & $7.80 \pm 1.36$ & $5.75 \pm 0.97$ & $<0.001^{*}$ \\
\hline 4. The report allows to extract relevant findings easily & $7.80 \pm 0.60$ & $8.95 \pm 0.22$ & $<0.001^{*}$ & $7.05 \pm 1.10$ & $7.80 \pm 0.83$ & $0.016^{*}$ \\
\hline 5. The structure of the report helps in finding relevant information quickly & $7.60 \pm 0.60$ & $9.10 \pm 0.31$ & $<0.001^{*}$ & $7.10 \pm 0.97$ & $8.05 \pm 0.89$ & $0.005^{*}$ \\
\hline 6. The report contains all relevant information & $8.60 \pm 0.75$ & $8.90 \pm 0.56$ & 0.113 & $6.80 \pm 1.58$ & $7.95 \pm 1.05$ & $0.009^{*}$ \\
\hline 7. The report allows an explanation of the patient's clinical symptoms & $8.45 \pm 0.69$ & $9.00 \pm 0.32$ & $0.003^{*}$ & $7.30 \pm 1.38$ & $7.50 \pm 0.51$ & 0.881 \\
\hline $\begin{array}{l}\text { 8. The report contains all information necessary for subsequent patient man- } \\
\text { agement }\end{array}$ & $7.90 \pm 0.79$ & $8.85 \pm 0.37$ & $<0.001^{*}$ & $6.65 \pm 1.42$ & $8.60 \pm 0.82$ & $<0.001^{*}$ \\
\hline $\begin{array}{l}\text { 9. I have no open questions, a further consultation with the radiologist is not } \\
\text { necessary }\end{array}$ & $8.55 \pm 0.76$ & $9.00 \pm 0.32$ & $<0.001^{*}$ & $6.25 \pm 1.45$ & $6.75 \pm 1.74$ & 0.386 \\
\hline 10. The report explains the MR images well & $8.35 \pm 0.74$ & $9.05 \pm 0.39$ & $0.020^{*}$ & $7.10 \pm 0.93$ & $7.95 \pm 0.75$ & $0.003^{*}$ \\
\hline 11. Please evaluate the overall quality of the report on a scale from 1 to 5 & $3.80 \pm 0.41$ & $4.0 \pm 0.00$ & $0.037^{*}$ & $3.60 \pm 0.50$ & $3.85 \pm 0.49$ & 0.127 \\
\hline 12. Favored report type [\%; n] & $15(3 / 20)$ & $85(17 / 20)$ & NA & $45(9 / 20)$ & $55(11 / 20)$ & NA \\
\hline
\end{tabular}

regarding clarity $(\mathrm{p}<0.001)$, comprehension $(\mathrm{p}<0.001)$, apprehension of relevant findings quickly $(\mathrm{p}=0.016)$, and the structure of the report in helping to find relevant information quickly $(\mathrm{p}=0.005)$. Furthermore, the satisfaction with the content in regard to completeness of information necessary for immediate subsequent patient management $(\mathrm{p}<0.001)$ and the explanation of the MR images were evaluated significantly higher for the SR by both raters $(p=0.020)$. Rater 1 stated that SR allow for a better explanation of the patient's clinical symptoms $(p=0.003)$ and significantly reduce the number of open questions, which would have implied further consultation with the radiologist ( $\mathrm{p}<0.001$ ). Furthermore, Rater 2 had the opinion that the SR contained all relevant information ( $\mathrm{p}<0.009)$. Rater 1 did not find the length of the two sorts of reports to be different, Rater 2 found the length of SR significantly more appropriate $(\mathrm{p}<0.001)$.

The overall quality of the report was rated in favor of the SR in case of Rater $1(\mathrm{p}=0.004)$ and, at least in tendency, in the case of Rater 2 (Table 3). The favored sort of report was the SR for both raters.

\section{Discussion}

This study showed that SR substantially increase the report quality of brain MRI after MT in patients with AIS compared to FTR. To the best of our knowledge, no previous studies have analyzed this challenging issue. The data presented in this manuscript indicate that the use of SR leads to significantly improved report quality, completeness, and readability. These findings are consistent with previous studies, which have reported a superior

Results are depicted as mean values with standard deviation for Questions 1 to 11 and as percentage for question 12

Significance was assumed if $\mathrm{p}<0.05$ and highlighted by using italics

FTR free text report, SR structured report, NA not applicable

* $p<0.05$ 
report quality of SR in a number of diagnostic modalities and clinical settings $[10,14,15]$. One advantage of the SR developed in this study was that also small structures with high clinical impact for clinicians, as for example the internal capsule, are often not mentioned in FTR.

In ischemic stroke, the patterns of infarction, and possible complications, such as hemorrhage, infarct swelling or, brain tissue herniation, may be challenging to detect. The accurate diagnosis of the stroke patients post recanalization status is of high importance because it is essential for determining the correct treatment approach and risk stratification for further rehabilitation management. MRI is the imaging modality of choice for precise imaging of brain tissue and ischemia. Thus, physicians in charge of stroke care depend on high quality MRI reports. It has been shown for other diseases that SR can increase the report quality and, thereby, make the work of referring clinicians easier [15]. Besides a thorough clinical examination, accurate reporting is important for being able to provide the highest standards in diagnostics and therapy.

The evaluation results of the neurologist raters in this study showed that SR was significantly favored over the FTR regarding report clarity, comprehension, apprehension of relevant findings quickly, and structure. Furthermore, satisfaction with necessary information for the immediate subsequent patient management, explanation of the MR images as well as the overall favored sort of report was rated in favor of SR. These superior results of the SR over the FTR have been previously reported and discussed in a variety of other clinical fields [12, 14-16]. For example, SR of MRI in patients with primary rectal cancer achieved significantly higher satisfaction rates with report content and clarity as well as overall report quality in comparison to FTR. Potential benefits for surgical planning and interdisciplinary communication were deduced from this [15].

Furthermore, in our study the neurologists had less open questions resulting in a reduced need to consult the reporting radiologists. This may have a positive effect on the workflow on both sides.

The educational aspects of SR for radiology residents in training by, for example, containing all key features with correct wording have been elaborated before [17], which further underlines the importance of SR implementation in stroke care.

In this work, SRs were created by using a software tool that translates clickable decisions into predefined text phrases.

This study has several limitations. First, the retrospective design may have introduced some unexpected bias relative to the radiologist who interpreted the examinations prospectively. Only two clinicians were involved in the review of SR vs. FTR. However, we tried to minimize this confinement by the strict independence of our reviewers. As the reviewers were not involved in the study design at the beginning, this may minimize a bias towards one or the other type of reporting. Furthermore, we did not measure the time needed for producing the SR and FTR was not included, simply because the study was retrospective and times for FTR generation were not available. Further studies should investigate the impact of SR in stroke MRI on clinical practice and workflows prospectively.

\section{Conclusions}

This study suggests that structured reports compared to free-text reports of brain MRI examinations in acute ischemic stroke patients which have been treated using mechanical thrombectomy is favored by stroke neurologists regarding clarity, comprehensibility, quick apprehension of relevant findings and the structure of the report. Structured reports potentially facilitate the stroke teams planning regarding next treatment steps and may lead to a higher satisfaction level of referring neurologists.

\section{Abbreviations}

AIS: Acute ischemic stroke; Art. TOF: Arterial time-of-flight MR angiography; AT: Acquisition time; CT: Computer tomography; CTA: CT angiography; DWl: Diffusion weighted imaging; FLAIR: Fluid attenuation inversion recovery; FTR: Free-text report; MR: Magnetic resonance; MRI: Magnetic resonance imaging; MT : Mechanical thrombectomy; NA: Not applicable; NIHSS: National Institutes of Health Stroke Scale; SR: Structured report; T: Tesla; TE:Time to echo; TR: Time to repeat; TI: Inversion time.

\section{Acknowledgements}

Not applicable.

\section{Authors' contributions}

S.M. and D.M.H made substantial contributions to the conception and design of the work, the acquisition, analysis, and interpretation of data and have drafted the work or substantively revised it. K.B. and B.I. made substantial contributions to the acquisition of data and by rating the SR and FTR as well as interpreting the data and revising the manuscript. T.A. made substantial contributions to the creation of new SR software used in the work. C.Z. and B.F. made substantial contributions to the conception and design of the work and revised the manuscript. All authors have read and approved the final manuscript.

\section{Funding}

Open Access funding enabled and organized by Projekt DEAL.

\section{Availability of data and material}

The datasets used and/or analysed during the current study are available from the corresponding author on reasonable request.

\section{Declarations}

Ethics approval and consent to participate

Informed consent was waived due to the retrospective nature of the study by the ethics committee of the Technical University of Munich. All procedures were approved by the ethics committee of the Technical University of Munich 
(application number 56/20 S-KH). All methods were carried out in accordance with the Declaration of Helsinki.

\section{Consent for publication}

Not applicable.

\section{Competing interests}

BF: Consultant for Stryker, speaker honoraria for Medtronic, Research grant from ischemaView. The other authors declare that they have no competing interests.

\section{Author details}

${ }^{1}$ Department of Diagnostic and Interventional Neuroradiology, Klinikum Rechts Der Isar, School of Medicine, Technical University Munich, Ismaninger Straße 22, 81675 Munich, Germany. ${ }^{2}$ Department of Radiology, University Hospital, LMU Munich, Munich, Germany. ${ }^{3}$ Department of Neurology, Klinikum Rechts Der Isar, School of Medicine, Technical University Munich, Munich, Germany.

Received: 7 March 2021 Accepted: 17 May 2021

Published online: 25 May 2021

\section{References}

1. Feigin VL, Lawes CM, Bennett DA, Barker-Collo SL, Parag V. Worldwide stroke incidence and early case fatality reported in 56 population-based studies: a systematic review. Lancet Neurol. 2009;8:355-69.

2. Berkhemer OA, Fransen PS, Beumer D, van den Berg LA, Lingsma HF, Yoo $\mathrm{AJ}$, et al. A randomized trial of intraarterial treatment for acute ischemic stroke. N Engl J Med. 2015;372:11-20.

3. Campbell BC, Mitchell PJ, Kleinig TJ, Dewey HM, Churilov L, Yassi N, et al. Endovascular therapy for ischemic stroke with perfusion-imaging selection. N Engl J Med. 2015;372:1009-18.

4. Goyal M, Demchuk AM, Menon BK, Eesa M, Rempel JL, Thornton J, et al. Randomized assessment of rapid endovascular treatment of ischemic stroke. N Engl J Med. 2015;372:1019-30.

5. Jovin TG, Chamorro A, Cobo E, de Miquel MA, Molina CA, Rovira A, et al. Thrombectomy within 8 hours after symptom onset in ischemic stroke. N Engl J Med. 2015;372:2296-306.

6. Saver JL, Goyal M, Bonafe A, Diener HC, Levy El, Pereira VM, et al. Stentretriever thrombectomy after intravenous t-pa vs. T-pa alone in stroke. N Engl J Med. 2015;372:2285-95.
7. Winstein CJ, Stein J, Arena R, Bates B, Cherney LR, Cramer SC, et al. Guidelines for adult stroke rehabilitation and recovery: a guideline for healthcare professionals from the american heart association/american stroke association. Stroke. 2016;47:e98-169.

8. Ganeshan D, Duong PT, Probyn L, Lenchik L, McArthur TA, Retrouvey M, et al. Structured reporting in radiology. Acad Radiol. 2018;25:66-73.

9. Pinto Dos Santos D, Brodehl S, Baessler B, Arnhold G, Dratsch T, Chon $\mathrm{SH}$, et al. Structured report data can be used to develop deep learning algorithms: a proof of concept in ankle radiographs. Insights Imaging. 2019;10:93

10. Dickerson E, Davenport MS, Syed F, Stuve O, Cohen JA, Rinker JR, et al. Effect of template reporting of brain mris for multiple sclerosis on report thoroughness and neurologist-rated quality: results of a prospective quality improvement project. J Am Coll Radiol. 2017;14(371-379):e371.

11. Lin E, Powell DK, Kagetsu NJ. Efficacy of a checklist-style structured radiology reporting template in reducing resident misses on cervical spine computed tomography examinations. J Digit Imaging. 2014;27:588-93.

12. Suh-Burgmann EJ, Flanagan T, Lee N, Osinski T, Sweet C, Lynch M, et al. Large-scale implementation of structured reporting of adnexal masses on ultrasound. J Am Coll Radiol. 2018;15:755-61.

13. Larrue V, von Kummer RR, Muller A, Bluhmki E. Risk factors for severe hemorrhagic transformation in ischemic stroke patients treated with recombinant tissue plasminogen activator: a secondary analysis of the european-australasian acute stroke study (ecass ii). Stroke. 2001;32:438-41.

14. Gassenmaier S, Armbruster M, Haasters F, Helfen T, Henzler T, Alibek S, et al. Structured reporting of mri of the shoulder-improvement of report quality? Eur Radiol. 2017;27:4110-9.

15. Norenberg D, Sommer WH, Thasler W, D'Haese J, Rentsch M, Kolben $T$, et al. Structured reporting of rectal magnetic resonance imaging in suspected primary rectal cancer: Potential benefits for surgical planning and interdisciplinary communication. Invest Radiol. 2017;52:232-9.

16. Bosmans JM, Weyler JJ, De Schepper AM, Parizel PM. The radiology report as seen by radiologists and referring clinicians: results of the cover and rover surveys. Radiology. 2011;259:184-95.

17. Collard MD, Tellier J, Chowdhury AS, Lowe LH. Improvement in reporting skills of radiology residents with a structured reporting curriculum. Acad Radiol. 2014;21:126-33.

\section{Publisher's Note}

Springer Nature remains neutral with regard to jurisdictional claims in published maps and institutional affiliations.
Ready to submit your research? Choose BMC and benefit from:

- fast, convenient online submission

- thorough peer review by experienced researchers in your field

- rapid publication on acceptance

- support for research data, including large and complex data types

- gold Open Access which fosters wider collaboration and increased citations

- maximum visibility for your research: over $100 \mathrm{M}$ website views per year

At BMC, research is always in progress.

Learn more biomedcentral.com/submissions 